\title{
Violencia en el noviazgo y su relación con la dependencia emocional pasiva en estudiantes universitarios
}

\section{Violence in the courtship and their relationship with the passive emotional dependence in university students}

Arturo Del Castillo Arreola

María Esther Hernández Díaz

Angélica Romero Palencia

Scarlett Iglesias Hoyos

Universidad Autónoma del Estado de Hidalgo

\section{Resumen}

El noviazgo es una etapa donde las personas tienen la oportunidad de conocerse, aprender a compartir, identificar afinidades e, inclusive, detectar indicios de violencia en la relación a largo plazo. Éstos últimos pueden aumentar en frecuencia e intensidad con el paso del tiempo, hasta representar un precedente de la violencia doméstica. Estudios previos refieren que la presencia de ansiedad por separación como indicador de dependencia emocional en las relaciones interpersonales ilustra las variables asociadas al origen de la violencia en el noviazgo. El objetivo del presente estudio fue identificar la relación entre los factores de dependencia emocional y violencia en el noviazgo entre estudiantes universitarios. Participaron 317 estudiantes de las licenciaturas en Medicina, Administración y Derecho de la Universidad Autónoma del Estado de Hidalgo. Se utilizó el Cuestionario de Dependencia Emocional Pasiva y el Cuestionario de Violencia en el Noviazgo, para evaluar las variables mencionadas. Se llevó a cabo un análisis de correlación de Pearson, con el cual se determinaron relaciones positivas, estadísticamente significativas, entre los factores ansiedad por separación y expresiones límite; del cuestionario de dependencia se encontraron relaciones positivas, estadísticamente significativas, con el chantaje, el control y los celos. A partir de estos resultados, se podrán plantear intervenciones cuyo principal objetivo sea desarrollar habilidades sociales que faciliten la independencia y la mejoría en las relaciones de noviazgo.

Palabras clave: violencia en el noviazgo, dependencia emocional, estudiantes universitarios, ansiedad por separación, expresiones límite.

Nota del autor

Arturo Del Castillo Arreola, Instituto de Ciencias de la Salud (ICS), Universidad Autónoma del Estado de Hidalgo (UAEH); María Esther Hernández Díaz, ICS, UAEH; Angélica Romero Palencia, ICS, UAEH; Scarlett Iglesias Hoyos, ICS, UAEH.

La correspondencia en relación con este artículo debe dirigirse a Arturo Del Castillo Arreola, ICS, UAEH, Ex hacienda La Concepción, carretera Pachuca-Tilcuautla, C. P. 42060, tel. (771) 7172000 ext. 5104 y 5118, Tilcuautla, Hidalgo, México.

Dirección electrónica: artur78@yahoo.com 


\begin{abstract}
The courtship is a stage where people have the opportunity to meet each other, to learn how to share, to identify similarities and, inclusive, to detect symptoms of dating violence in the long term relationship. These last they can increase in frequency and intensity with the step of the time, until it represents a precedent of the domestic violence. Previous studies refer that the presence of separation anxiety as a sing of emotional dependency and deficiency is some factor that is associated with the genesis of dating violence. The objective of the present study was to identify the relationship between the factors of emotional dependence and violence in the courtship among university students. The sample included 317 doctor, administration and law students of the University of the State of Hidalgo. It was used the Emotional Dependence Questionnaire and Dating Violence Questionnaire, to evaluate the mentioned variables. Pearson correlation analysis was used, obtaining statistically significant positive relations between the separation anxiety and border expression factors of the Emotional Dependency questionnaire and the emotional blackmail, control and jealous factors of the dating violence questionnaire. Based on these results, psychological interventions could be planned with the objective of developing social skills that facilitate the independency and improvement of interpersonal relationships.

Keywords: dating violence, emotional dependency, college students, separation anxiety, selfaggression expressions.

En los últimos años la violencia en las relaciones de pareja ha sido considerada un problema de salud pública; no respeta clase social, nivel educativo, religión, sexo, edad, ni raza (Amaro, 2011; Ramírez-Rivera \& NúñezLuna, 2010). En México, este fenómeno ha sido de gran interés, según inferimos de la atención recibida no sólo por parte de investigadores, sino también por instituciones públicas, privadas y ONG, encargadas de prevenirlo, así como de atender sus efectos mediante programas de atención secundaria y terciaria. No obstante, se ha otorgado mayor prioridad a erradicar la violencia de pareja, ejercida contra las mujeres, con base en un modelo clínico de hombreagresor y mujer-víctima, sin tomar en cuenta la dinámica de las relaciones interpersonales desde el noviazgo (Rojas-Solís, 2013).

Corral (2009) menciona que la violencia de pareja en jóvenes no ha recibido tanta atención en la literatura como la violencia en parejas adultas, sin embargo, la violencia durante la relación de noviazgo puede ser un pronóstico de violencia doméstica. Dicha violencia inicial se refiere a la serie de actos que lastiman a otra persona, en el contexto de una relación de atracción y citas (Close, 2005), o bien, a todo ataque intencional, omisión, actitud o expresión que genere daño emocional, físico o sexual en una relación de noviazgo. Aun cuando no se determina según la edad, hace referencia a parejas de novios adolescentes y adultos jóvenes que no conviven en una relación marital (Rubio-Garay, LópezGonzález, Saúl, \& Sánchez-Elvira-Paniagua, 2012). A nivel internacional, los estudios sobre la violencia en relaciones amorosas de jóvenes
\end{abstract}


universitarios indican que este fenómeno afecta a parte importante de esta población (Organización Mundial de la Salud [OMS], 2011), como lo refiere un estudio realizado en Sudáfrica, donde $42 \%$ de las mujeres y $38 \%$ de los hombres refirieron haber sido víctimas de violencia física en el noviazgo (OMS, 2012).

En lo que respecta a la situación en México, según la Secretaría de Seguridad Pública (SSP, 2012), tres de cada diez jóvenes de entre 15-29 años indicaron haber sufrido violencia durante el noviazgo. El 41.9 \% declaró que había tenido al menos un episodio de violencia como insultos, burlas y críticas en su entonces actual noviazgo; el $27.3 \%$ fue víctima de agresiones físicas como empujones, patadas y bofetadas por parte de su novio(a); el $23.8 \%$ experimentó amenazas; y $20.7 \%$ fue obligado(a) a tener relaciones sexuales o a realizar actividades que no le gustaban en la intimidad (Instituto Nacional de Estadística, Geografía e Informática [INEGI], 2013).

Respecto a la violencia contra las mujeres, los índices son alarmantes, pues de acuerdo con la Encuesta Nacional de las Dinámicas de las Relaciones en los Hogares (INEGI, 2011), $43.2 \%$ de las mujeres mayores de 15 años en el país ha sido víctima de esta situación. Por lo que respecta al estado de Hidalgo, dieciseisavo lugar en la lista de estados donde las mujeres reportan más episodios de violencia, 59 de cada 100 mujeres manifestaron haber padecido algún incidente de violencia de tipo emocional, expresada a través de amenazas, menosprecios y prohibiciones; dicho tipo de violencia prevalece en el estado (INEGI, 2010, 2011).
Se ha encontrado que la violencia en el noviazgo se relaciona con diferentes variables como la baja autoestima en el agresor(a), conductas celotípicas, carencia de empatía (Adams \& Cervantes, 2012; Olvera, Arias, \& Amador, 2012), uso excesivo de alcohol y experiencias de violencia durante la infancia que conllevan a la aceptación y tolerancia de actos violentos (Dejonghe, Bogart, Levendosky, Von Eye, \& Davidson, 2005; OMS, 2012; Pérez, 2012). Así también, dentro de las variables que se han asociado a la aparición de este tipo de violencia se encuentra la dependencia emocional (Oropeza, 2011), referida a una demanda persistente de necesidades afectivas que un individuo pretende cubrir de manera desadaptada con otras personas; se caracteriza por creencias sobrevaloradas sobre la amistad, intimidad e interdependencia (Castello, 2011).

Anteriormente, se consideraban personas dependientes a aquellas que se mostraban sumisas, necesitadas de afecto; empero, hoy por hoy algunos autores (Castello, 2011; Mansukhani, 2013; Tiscareño \& Velázquez, 2011), han descrito diversos tipos de dependencia emocional en las relaciones interpersonales:

1. Dependencia emocional de tipo convencional (pasiva o sumisa). Se refiere a las personas subordinadas y sumisas, que hacen lo que el otro dice; capaces de aceptar humillaciones, le atribuyen características idealizadas, pues son cualidades que le hacen falta como el amor y la confianza en sí misma.

2. Dependencia con vinculación oscilatoria. Hace referencia a las personas que 
presentan características de dependencia sólo por un periodo de tiempo; la persona evita tener pareja después de pasar por la época de la dependencia hasta que resurge la necesidad de tener afecto.

3. Dependencia emocional dominante. Describe a personas que implantan temor en la pareja para que no las abandone. Se domina a la pareja mediante menosprecio, explotación e imposición. Tienen el poder en la relación, satisfacen así su necesidad afectiva.

La dependencia emocional de tipo pasivo o sumiso es más frecuente en las mujeres y está caracterizada por la ansiedad (Mansukhani, 2013). La persona siente temor de ser abandonada o rechazada y adopta un papel sumiso en las relaciones interpersonales con el fin de evitar el rechazo y/o abandono, por tanto, es proclive a estar en relaciones abusivas, ante su dificultad para terminar una.

La dependencia emocional pasiva se caracteriza por excesivas demandas afectivas y estrechas, baja valoración de sí mismo, idealización de la pareja, necesidad imperiosa del otro, establecimiento de relaciones desequilibradas, abandono o modificación de planes propios para satisfacer a los otros, miedo excesivo a la soledad y al desamparo, deseos de protección, desconfianza, comportamientos de sumisión, pensamientos obsesivos en torno a la pareja, celos, búsqueda de seguridad, exclusividad sexual y afectiva, además de creencia en los mitos o estándares románticos de la sociedad (Méndez, Favila, Valencia, \& Díaz-Loving, 2012; Tiscareño \& Velázquez, 2011).
Arellano (2010) menciona que las personas con altos niveles de dependencia emocional pasiva tienen dificultad para expresar ciertos tipos de sentimientos (dolor, amor, rabia, miedo, etc.); no se dejan conocer fácilmente, sólo con aquellos que se consideran seguros; sienten la necesidad de complacer; comprometen sus propios valores e integridad; por ello, no saben decir "no", y si lo hacen se sienten culpables. A menudo personas con rasgos de dependencia tienen relaciones sexuales cuando en realidad no querían, piensan que hacer cosas para sí mismos es egoísta y anteponen necesidades de los demás a las propias, hacen lo que su pareja o amigos quieren que hagan en vez de lo que ellas quieren y no expresan sus verdaderos sentimientos porque les preocupa la reacción de los demás.

Respecto a sus relaciones, creen en el amor a primera vista, la gente que es agradable con ellos les resulta aburrida, piensan que sus problemas se resolverán si consiguen que sus parejas cambien, se sienten incompletos sin pareja, creen que los demás pueden hacerles felices, se sienten responsables de los sentimientos de otros. Asimismo, perciben la soledad con pánico, enojo y desesperación, tienen una necesidad de acercamiento constante hacia la pareja, hacen llamadas y mandan mensajes continuamente, existe el deseo de hacer con la otra persona cualquier actividad. Se presenta, por un lado, la necesidad emocional, y por otro, se sufre la ansiedad ante la idea de una posible pérdida del otro. Existe una paradoja: desean pero temen la cercanía, ya que realmente desean amar y ser amados, aunque su parte dependiente le teme demasiado al acercamiento por el dolor 
y rechazo que puede causar, además, carecen de habilidades sociales (Arellano, 2010; Echeverría, 2008).

La dependencia emocional pasiva se ha relacionado con la aparición de daños físicos y emocionales, ya que estas personas creen que amar es ofrecerlo todo, por tanto, justifican las conductas de la pareja de manera idealizada y suelen tener celos irreales ante la ansiedad de perderle. Su autoestima disminuye y se aferran a la relación, sin ser capaces de terminarla (Massa, Pat, Keb, Canto, \& Chan, 2011). Según Méndez et al. (2012), la dependencia emocional pasiva engloba dos factores principales:

1. Ansiedad por separación. Engloba las expresiones de miedo, preocupación, ansiedad o angustia excesivas que se producen ante la posibilidad de disolver la relación, debido a distanciamientos temporales que activan pensamientos de abandono y de soledad.

2. Expresiones límite. Describe expresiones o conductas que anteceden o que son impulsos de autoagresión, que son vistas como estrategias de aferramiento para evitar la pérdida.

El estudio de la dependencia emocional se ha abordado desde diferentes perspectivas teóricas. Según el psicoanálisis, las experiencias tempranas juegan un papel importante en la constitución del individuo. Freud (1905) asevera que existen etapas psicosexuales, una de ellas es la etapa oral, cuando el niño busca el placer por la boca, su satisfacción puede estar restringida por quienes lo cuidan; la independencia del niño debe funcionar conforme a ciertos límites. Las personas que tienen una fijación en esta etapa presentan trastornos en el recibir y el tomar; mientras el recibir fallido puede tomar la forma de dependencia pasiva, el tomar fallido puede resultar en envidia y avaricia.

En base en lo anterior, Balint (1968) hablaba de la falla básica: se experimenta cuando alguien ha defraudado a la persona, por un cuidado deficiente, ansioso, rígido, fuera de tiempo, sobre estimulado o simplemente incomprendido e indiferente. Esta falla es la precursora de las relaciones objetales posteriores y de la aparición de dos tipos de carácter: ocnofilia y filobatismo. El primero se refiere a la tendencia a sentir inseguridad, a mantener una unión muy estrecha con los objetos, por lo cual se contempla la separación como la peor amenaza. El segundo se refiere a una tendencia a separarse de los objetos, a vivir la cercanía como un peligro de ser atrapado, desarrollar formas de manipular a los objetos sin involucrarse profundamente (Cueli, Reidl, Martí, Lartigue, \& Michaca, 2009). Como se puede observar, la ocnofilia es un rasgo que los dependientes emocionales presentan, ya que temen la disolución de la relación de pareja.

Por otro lado, Kohut (1959) menciona que para la adquisición de la autonomía era necesaria la intervención real de los padres o personas significativas (objetos del self), mediante la empatía con el fin de concentrarse acerca de sus necesidades y sus deseos de elogios: éstos alimentan su narcicismo y su omnipotencia, dado que se idealiza al objeto, que le debe servir de modelo a seguir. Tal función idealizadora se cumple y se incorpora mediante la internalización. Cuando no existe 
dicha función, se produce baja autoestima, deseos de agradar, vulnerabilidad ante las críticas, anhelos profundos de interés y afecto por parte de los demás, como en el caso de las personas dependientes emocionalmente.

Desde los modelos derivados del aprendizaje social, la dependencia emocional se entiende como un patrón conformado por aspectos cognitivos, emocionales y conductuales que presenta un individuo, enfocados hacia otra persona como fuente de satisfacción y seguridad personal. Esto trae consigo creencias erróneas acerca del amor y de la vida en pareja: conllevan pensamientos irracionales, no se puede concebir la vida si no es dentro de una relación, aunque ésta no sea muy satisfactoria. Existe una carencia afectiva temprana y una focalización excesiva en fuentes externas de la autoestima (Méndez et al., 2012).

Las carencias afectivas hacen referencia a las personas con dependencia emocional que han sufrido una grave carencia de afecto desde el principio de su vida, sin personas que las hayan querido o estado pendientes de ellas, presentan abuso físico o psicológico, por lo tanto, una persona significativa caracterizada por ser fría u hostil le resultará más familiar. Sus esquemas personales se forjan con base en creencias negativas sobre sí mismas, consideran que los demás son superiores a ellas y que, por lo tanto, necesitan ayuda y afecto de los demás (Lemos, Jaller, González, Díaz, \& De la Ossa, 2012; Tiscareño \& Velázquez, 2011).

El mantenimiento de la vinculación y focalización excesiva en fuentes externas de autoestima, se refiere a que el dependiente emocional se centra demasiado en los demás, se desatiende a sí mismo y despliega estrategias de sumisión y entrega para ser aceptado por los demás. Este vínculo es más difícil de experimentar en las mujeres, ya que ellas tienen una mayor disposición empática y compasiva durante el contacto social que los varones, misma que condiciona la aparición de la dependencia emocional (Jaller \& Lemos, 2009; Tiscareño \& Velázquez, 2011).

Desde una perspectiva etnopsicológica, en el caso de México, domina una cultura colectivista que lleva a las personas a buscar ser empáticas con los demás, de tal manera que se enfocan en las necesidades de los otros hasta llegar a ser interdependientes; es decir, estar pendiente del otro para socializar, en algunos casos, estimula el desarrollo de la dependencia emocional. Por esto, según los factores socioculturales, las mujeres tienden a amar de una forma más entregada que los hombres, e incluso, existen estereotipos que les inculcan la idea de no poderse valer por ellas mismas, su vida depende de los hombres (Rivera \& Díaz- Loving 2002).

Con respecto a la asociación entre la dependencia emocional y la violencia en el noviazgo, un estudio realizado en México por Oropeza (2011) encontró que, en el caso de las mujeres, el temor a la separación, y, en los hombres, la necesidad de afecto, están relacionados con la violencia psicológica y sexual. Ambos grupos consideran que la pareja es la única que les brinda apoyo y prefieren dejar de realizar sus actividades diarias por estar con ella; además, explica que ambos sexos pueden mostrarse como per- 
sonas sumisas al utilizar la súplica, la influencia social y el autodesprecio, susceptibles a la violencia que ejerce su pareja.

A partir de tales antecedentes, el objetivo del presente estudio fue determinar la relación existente entre los factores de la dependencia emocional pasiva y la violencia en el noviazgo en estudiantes universitarios de forma general y por sexo. La hipótesis alterna nos indica que se encontrarán correlaciones positivas, estadísticas significativas entre las dimensiones de violencia en el noviazgo y dependencia emocional pasiva en lo estudiantes universitarios de la UAEH. Con respecto al sexo, en el caso de las mujeres se espera que a mayor presencia de ansiedad por separación y expresiones límite, mayor será el riesgo de recibir violencia de tipo verbal, así como chantaje, celos, humillación y control en las relaciones de noviazgo.

\section{Método}

\section{Tipo de diseño y estudio}

Se llevó a cabo un diseño no experimental, transversal, correlacional.

\section{Participantes}

Participaron 317 estudiantes de la UAEH, $165(52.1 \%)$ eran mujeres y $152(47.9 \%)$ hombres, fueron seleccionados mediante un muestreo no probabilístico. De la muestra total, $108(34.1 \%)$ pertenecían a la licenciatura en Administración, 89 (28.1 \%) de Derecho y $120(37.9 \%)$ de Medicina. Con respecto a la distribución por relación de pareja, 162 (51.1\%) eran novios, 19 (6.0\%) tenían "amigovios(as)", $15(4.7 \%)$ mantenían una relación free, y 10
(3.2\%) estaban en otro tipo de relación que no especificaron. Todos los casos se incluyeron en los análisis.

\section{Instrumentos}

Violencia en el noviazgo: Se utilizó el Cuestionario de Violencia en el Noviazgo construido y validado por García y Guzmán (2014). El cuestionario está compuesto por 52 reactivos que se distribuyen en siete factores de la violencia en el noviazgo: 1) Violencia Verbal, 2) Chantaje, 3) Control, 4) Violencia Social, 5) Exigencias, 6) Celos y Conflicto y 7) Humillación, con exclusión de la violencia física. En una escala tipo Likert con cinco opciones de respuesta. Cuenta con un Alfa de Cronbach, $\alpha=0.978$.

El factor Violencia Verbal incluye reactivos como: "Me amenaza con irse con otra (o)", "Me compara con parejas pasadas", "Me ha dicho que soy feo (a) o poco atractivo (a)", "Me ha dicho que estoy gorda (o)".

El factor Chantaje incluye los reactivos: "Es posesivo", "Cambia de humor de manera brusca", "Provoca cambios en mi forma de vestir", "Explota por cualquier cosa", "Llora para hacerme sentir mal".

En el factor Celos y Conflicto están: "Lee mis correos electrónicos para enterarse de mi vida", "Me cela todo el tiempo", "Se enoja si me ve platicando con amigos del sexo opuesto", "Quiere ir conmigo a todas partes".

El factor Control incluye los reactivos: "Constantemente me manda mensajes al celular para saber dónde estoy”, "Controla las llamadas de mi celular", "Controla mis actividades", "Me amenaza con abandonarme". 
En el factor Violencia Social están los reactivos: "Me quita mi celular para leer los mensajes que me envían otras personas", "Me prohíbe vestir como me gusta", "Me insulta", "Me impide tener muchos amigos", "Me impide tener amigos del sexo opuesto", "Me exige que le ponga crédito a su celular para que me llame".

En el factor Exigencias se incluyen los reactivos: "Me exige que le dé la prueba de amor", "Me exige que pague todos nuestros gastos como pareja", "Me exige que le compre cosas costosas".

En el factor Humillación están: "Me humilla frente a otros", "No confía en mí", "Piensa que lo(a) estoy engañando", "Me reclama que pudiera serle infiel".

Dependencia emocional pasiva: Se evaluó mediante el Cuestionario de Dependencia Emocional Pasiva adaptado en México por Méndez, et al. (2012), originalmente construido y validado por Lemos y Londoño (2006). La versión adaptada está conformada por diez reactivos, agrupados en dos factores: 1) Ansiedad de Separación y 2) Expresiones Límite. Tiene una confiabilidad Alfa de Cronbach $(\alpha)=0.90$.

Dentro del factor Ansiedad por Separación se incluyen los reactivos: "Cuando discuto con $\mathrm{mi}$ pareja me preocupa que deje de quererme", "Si tengo planes y mi pareja aparece los cambio por estar con ella", "No tolero la soledad".

El factor Expresiones Límite está compuesto por los reactivos: "Me divierto sólo cuando estoy con mi pareja", "Me he hecho daño físicamente con la intención de que mi pareja no me abandone o que regrese a mi lado".

\section{Procedimiento}

Después de haber obtenido permiso de las autoridades correspondientes e invitado a los participantes, se acudió a diferentes grupos, desde primero hasta noveno semestre y se les explicó el objetivo de la investigación. El proceso se inició cuando se entregó una ficha de datos, después se repartieron los cuestionarios y se leyeron las instrucciones de cada instrumento. Además, se solicitó llenar todos los ítems de las diferentes escalas.

\section{Análisis de datos}

Para realizar los análisis estadísticos y obtener la tablas correspondientes se empleó el programa SPSS 17.0. Se verificaron los datos y se eliminaron aquellos casos donde se carecía de información en el instrumento. Se empleó la prueba estadística de correlación productomomento de Pearson para el análisis de las variables.

\section{Resultados}

El objetivo general del presente estudio fue determinar la relación existente entre las dimensiones de dependencia emocional pasiva y violencia en el noviazgo en estudiantes universitarios. En la tabla 1 se describen los resultados de medias y desviaciones estándar de dichas variables.

En la tabla 2 se muestran los resultados del análisis de correlación de Pearson para conocer las relaciones entre los factores del Cuestionario de violencia en el noviazgo y el Cuestionario de Dependencia Emocional Pasiva. 
Tabla 1

Medidas de tendencia central y dispersión de las variables dependencia emocional pasiva $y$ violencia en el noviazgo

\begin{tabular}{llll}
\hline \multicolumn{1}{c}{ Variable } & Rango teórico & Media $\bar{X}$ & Desviación estándar \\
& & & \\
\hline Dependencia emocional & & 13.52 & 4.01 \\
Ansiedad por separación & $7-28$ & 4.46 & 1.44 \\
Expresiones límite & $3-12$ & & \\
Violencia en el noviazgo & & 10.04 & 2.84 \\
Verbal & $8-40$ & 8.06 & 3.52 \\
Chantaje & $5-25$ & 13.93 & 5.99 \\
Celos y conflictos & $8-40$ & 11.36 & 3.97 \\
Control & $7-35$ & 7.74 & 2.76 \\
Social & $6-30$ & 3.41 & 1.16 \\
Exigencias & $3-15$ & 7.00 & 2.82 \\
Humillación & $5-25$ & & \\
\hline
\end{tabular}

Tabla 2

Relación entre las dimensiones de dependencia emocional pasiva y violencia en el noviazgo

\begin{tabular}{lll}
\hline \multirow{2}{*}{ Violencia en el noviazgo } & \multicolumn{2}{l}{ Dependencia emocional } \\
\cline { 2 - 3 } & Ansiedad por separación & Expresiones límite \\
\hline Verbal & $.262^{* *}$ & $.112^{*}$ \\
Chantaje & $.171^{* *}$ & $.179^{* *}$ \\
Celos y conflicto & $.210^{* *}$ & $.121^{*}$ \\
Control & $.165^{* *}$ & $.113^{*}$ \\
Social & .104 & .064 \\
Exigencias & .106 & .065 \\
Humillación & $.192^{* *}$ & .065 \\
\hline
\end{tabular}

$* p=<.05 \quad * * p=<.01$ 
Se encontró una relación positiva, estadísticamente significativa, entre la ansiedad por separación y las dimensiones de violencia: verbal $(r=.262)$, chantaje $(r=.171)$, celos y conflicto $(r=.210)$, control $(r=165)$, humillación $(r=$ .192). Esto indica que a mayor ansiedad por separación, mayor será la violencia recibida en lo que se refiere a chantaje, celos y conflicto, violencia verbal, control y humillación.

De igual manera se encontraron relaciones positivas, estadísticamente significativas, entre dependencia emocional en su factor de expresiones límite y las dimensiones de violencia: verbal $(r=.112)$, chantaje $(r=.179)$, celos y conflicto $(r=.121)$ y control $(r=.113)$. Lo anterior indica que a mayor índice de expresiones límite o conductas de impulsos de autoagresión para aferrarse a la pareja, mayor será la violencia verbal, el chantaje, los celos y conflicto, además del control.

En la tabla 3 se exponen los resultados del análisis de correlación de Pearson para conocer las relaciones entre los factores del Cuestionario de Violencia en el Noviazgo y el Cuestionario de Dependencia Emocional Pasiva por sexo.

Tabla 3

Relación entre las dimensiones de dependencia emocional pasiva y violencia en el noviazgo por sexo ( $H=$ hombres, $M=$ mujeres $)$

\begin{tabular}{lllll}
\hline \multicolumn{5}{l}{ Dependencia emocional } \\
\hline Violencia en el noviazgo & \multicolumn{2}{l}{ Ansiedad por separación } & \multicolumn{2}{l}{ Expresiones límite } \\
\hline Verbal & $\mathrm{H}$ & $\mathrm{M}$ & $\mathrm{H}$ & $\mathrm{M}$ \\
Chantaje & .153 & $.375^{* *}$ & -.027 & $.196^{*}$ \\
Celos y conflicto & .101 & $.281^{* *}$ & $.165^{*}$ & .096 \\
Control & .146 & $.275^{* *}$ & .094 & .104 \\
Social & .153 & $.195^{*}$ & .122 & .046 \\
Exigencias & .021 & $.168^{*}$ & .043 & .058 \\
Humillación & .121 & $.191^{*}$ & .006 & .048 \\
\hline
\end{tabular}

En el caso de las mujeres, se encontraron relaciones positivas, estadísticamente significativas, entre la dimensión ansiedad por separación $\mathrm{y}$ todos los factores de violencia en el noviazgo, es decir, a mayor ansiedad por separación mayor violencia verbal, social, chantaje, celos y conflicto, control, exigencias y humillación. En lo que se refiere a la dimensión expresiones límite 
se encontró una relación estadísticamente significativa con la violencia verbal $(r=.196)$. En el caso de los hombres, se encontró una relación estadísticamente significativa en la dimensión expresiones límite y el chantaje $(r=.165)$.

\section{Discusión}

El propósito de la presente investigación fue determinar la relación entre las dimensiones de dependencia emocional pasiva y de violencia en el noviazgo en estudiantes de las licenciaturas en Administración, Derecho y Medicina de la UAEH.

Se determinó la relación entre los factores de la violencia en el noviazgo y la dependencia emocional pasiva, pues los resultados mostraron relaciones positivas, estadísticamente significativas: cuando las personas presentan ansiedad de separación y expresiones límite, también reciben violencia verbal, chantaje, control, celos y conflicto. Según la literatura del tema, una persona que es dependiente de forma emocional utiliza diversos recursos positivos o negativos para lograr que la pareja se quede al lado de ella, y así siga satisfaciendo sus necesidades afectivas (Fuentes, 2011).

Estos resultados son consistentes con la investigación de Oropeza (2011) en estudiantes universitarios, donde encontró que las personas preocupadas por la idea de ser abandonadas por su pareja y capaces de arriesgar su vida, reciben amenazas, agresiones y ofensas, control de sus llamadas y actividades, para asegurarse de conservar el amor de la pareja. Esto coincide, ya que cuando una persona depende emocionalmente justifica las conductas de la pareja de manera idealizada y suele tener celos irreales ante la ansiedad de perderle; por tanto, es proclive a recibir violencia; piensa y cree que la pareja es única y exclusivamente de su pertenencia. Es incapaz de terminar una relación violenta, debido a la importancia excesiva que adquiere antes de sentir amor (Massa, et al., 2011).

Desde la perspectiva etnopsicológica, estos resultados podrían deberse a que en México, como cultura que fomenta la dependencia a través de la abnegación y flexibilidad, es más importante para los mexicanos la satisfacción de las necesidades de los otros antes que las propias, al preferir la automodificación: "primero tú, luego yo", se posibilita así el desarrollo de la dependencia emocional, en la medida en que la (el) novia(o) cubra las necesidades del otro (Rivera \& Díaz-Loving, 2002). Las personas con dependencia emocional sienten responsabilidad por los sentimientos de los demás: perciben la soledad con pánico, enojo y desesperación; tienen una necesidad constante de cercanía, por consiguiente, realizan actividades para complacer al otro con el fin de evitar el abandono (Arellano, 2010).

Los resultados de la presente investigación coinciden con la literatura respecto al temor que las personas dependientes tienen a la separación, al tomar un rol pasivo y otorgar a un tercero una posición dominante sobre ellos, mostrándose complacientes y afectuosos; la intención de este comportamiento es que nadie los abandone (Sarason \& Sarason, 2006), se anteponen así los 
planes personales de la persona amada e incluso se presentan conductas límites o autodestructivas ante la posibilidad inminente de la ruptura (Lemos \& Londoño, 2006).

También se realizaron las correlaciones entre la dependencia emocional pasiva y la violencia en el noviazgo por sexo. En el caso de las mujeres de esta muestra, el factor ansiedad de separación está relacionado con las formas de violencia verbal, chantaje, control, exigencias, violencia social, humillación, celos y conflicto, mientras que el factor expresiones límite sólo se asoció a la violencia verbal. Según los resultados, esto pueden deberse a estereotipos de género, donde a las mujeres se les enseña a ser sumisas, menos competentes, más cálidas y emocionales que los hombres; por tal razón, censuran conductas consideradas "no propias de su género" en presencia de los hombres y en los lugares públicos. Cuando se trata de dar prioridad a situaciones personales, prefieren atender las necesidades del novio, por el temor a la pérdida o el riesgo de ser rechazada; se vuelven vulnerables a la violencia e, inclusive, la minimizan (Frieze, Parsons, Johnson, Ruble, \& Zellman, 1978).

Mejía (2010) menciona que las expectativas estereotipadas de género: la sumisión, baja asertividad, inseguridad, pasividad, diálogo, sensibilidad e intuición sitúan a la mujer en un grado de dependencia emocional pasiva frente al varón, y la convierten en víctima potencial de maltrato.

En el caso de los hombres, se encontró la relación entre el factor expresiones límite y el chantaje, esto puede deberse a que socialmente como hombres se les enseña que deben ser independientes, autosuficientes, sustentos económicos y trabajadores, de tal manera que si muestran su lado vulnerable hacia ellas, podrían ser rechazados por las mujeres (González \& Fernández de Juan, 2010). En este caso, deben ser capaces de realizar cualquier cosa para que su pareja no los abandone, permiten los cambios de humor y el llanto como forma de chantaje ante la posibilidad de disolver la relación (Montero, 2009; Chávez, 2013).

Como conclusión se aceptan las hipótesis alternas y basándonos en los resultados obtenidos en la presente investigación, el miedo a la pérdida contamina el vínculo afectivo en el noviazgo y lo vuelve sumamente vulnerable y patológico. Esta dependencia no sólo se explica a nivel de vínculos, sino que las representaciones sociales, como las creencias erróneas acerca del amor y de la vida en pareja, influyen para que se produzcan pensamientos irracionales: la persona no puede concebir la vida si no es dentro de una relación, aunque ésta no sea muy satisfactoria, a partir de premisas como la abnegación, la seguridad familiar, la preservación de la imagen pública, la no asertividad (Díaz-Loving, marzo, 2006; Cienfuegos \& Díaz-Loving, 2010). En dicho contexto, una persona fría u hostil le resulta más familiar, ya que esquemas personales se forjan en creencias negativas sobre sí misma (Méndez et al., 2012; Tiscareño \& Velázquez, 2011). 


\section{Sugerencias y limitaciones}

Como sugerencias para futuras investigaciones se propone incluir una muestra más amplia y llevar a cabo un muestreo probabilístico. También se sugiere incluir otras medidas para la medición de la violencia en el noviazgo que involucren las áreas física, sexual y económica. En futuras investigaciones se sugiere realizar correlaciones por carrera y análisis de diferencias por sexo para ampliar el conocimiento del tema.

\section{Referencias}

Adams, J., \& Cervantes, R. (2012). Violencia pasiva en mujeres universitarias. Un estudio exploratorio de las causas del deterioro de la autoestima. Psicología y Salud, 22(1) 133139.

Amaro, A. (2011). Violencia en las relaciones de noviazgo: Un contraste entre la zona rural y urbana en hombres y mujeres (Tesis de licenciatura inédita). Universidad Nacional Autónoma de México, México.

Arellano, T. (2010). Dependencia emocional en la pareja: Propuesta de un instrumento para su medición (Tesis de licenciatura inédita). Universidad Salesiana, México.

Balint, M. (1968). The basic fault: Therapeutic aspects of regression. London: Tavistock Publications.

Castello, J. (2011). La superación de la dependencia emocional. España: Ediciones Corona Borealis.
Chávez, P. (2013). Violencia en el noviazgo hacia el varón. (Tesina de licenciatura inédita). Universidad Nacional Autónoma de México, México.

Cienfuegos, Y., \& Díaz-Loving, R. (2010). Violencia en la relación de pareja. En R. Díaz-Loving \& S. Rivera-Aragón (Eds), Antología psicosocial de la pareja, clásicos y contemporáneos (pp. 647-687). México: MA Porrúa.

Close, S. M. (2005). Dating violence prevention in middle school and high school youth. Journal of Child and Adolescents Psychiatric Nursing, 18(1), 2-9.

Corral, S. (2009). Estudio de la violencia en el noviazgo en jóvenes universitarios: Cronicidad, severidad y mutualidad de las conductas violentas. Psicopatología Clínica Legal y Forense, 9, 29-48.

Cueli, J., Reidl, L., Martí, C., Lartigue, T., \& Michaca, P. (2009). Teorías de la Personalidad. México: Trillas.

Dejonghe, E. S., Bogart, G. A., Levendosky, A. A., Von Eye, A., \& Davidson, W. S. (2005). Infant Exposure to Domestic Violence Predicts Heightened Sensitivity to Adult Verbal Conflict. Infant Mental Health Journal, 26(3), 268-281.

Díaz-Loving, R. (marzo, 2006). Psicología del mexicano y su impacto en la violencia intrafamiliar. Conferencia invitada. Foro Las víctimas del delito y su abordaje en las ciencias sociales y del comportamiento, Comisión Nacional de los Derechos Humanos, México. 
Echeverría, N. (2008). Falta de apego, dependencia emocional en un grupo de apoyo a mujeres que viven una relación destructiva (Tesis de licenciatura inédita). Universidad Insurgentes, México.

Freud, S. (1905). On dreams. London: Hogarth Press.

Frieze, I. H., Parsons, J. E., Johnson, P. B., Ruble, D. N., \& Zellman, G. L. (1978). Women and Sex Roles. A social Psychology Perspective. USA: Norton and Company.

Fuentes, P. (2011). El impacto de la identidad de género, la autoestima y el apego en una relación de codependencia afectiva en el noviazgo. (Tesis de licenciatura inédita). Universidad Nacional Autónoma de México, México.

García, M., \& Guzmán, R. (2014). Validación de una escala de violencia en el noviazgo (Documento inédito). Universidad Autónoma del Estado de Hidalgo, Pachuca, Hgo.

González, H., \& Fernández de Juan, T. (2010). Género y maltrato: Violencia de pareja en los jóvenes de Baja California. Revista Estudios Fronterizos, 11(22), 97-128.

Instituto Nacional de Estadística, Geografía e Informática [INEGI]. (2010). Estadísticas a propósito del día internacional de la eliminación de la violencia contra la mujer. Datos de Hidalgo. Pachuca, México: Autor.

INEGI. (2011). Encuesta Nacional sobre la Dinámica de las Relaciones en los Hogares. México: Autor.
INEGI. (2013). Estadísticas a propósito del día internacional de la juventud: Datos nacionales. México: Autor.

Jaller, C., \& Lemos, M. (2009). Esquemas desadaptativos tempranos en estudiantes universitarios. Acta Colombiana de Psicología, 12(2), 77-83.

Kohut, H. (1959). Introspection, empathy, and psychoanalysis: An examination of the relationship between mode of observation and theory. Journal of the American Psychoanalytic Association, 7, 459-483.

Lemos, M., Jaller, C., González, A., Díaz, T., \& De la Ossa, D. (2012). Perfil cognitivo de la dependencia emocional en estudiantes universitarios en Medellín, Colombia. Universitas Psychologica, 11(2), 395-404.

Lemos, M., \& Londoño, N. (2006). Construcción y validación del cuestionario de dependencia emocional en población colombiana. Acta colombiana de psicología, 9(2), 127140.

Mansukhani, A. (2013). Dependencias interpersonales: Las vinculaciones patológicas. Conceptualización, diagnóstico y tratamiento. En A. García-Rojas \& F. Cabello-Santamaría (Eds), Actualizaciones en sexología clínica y educativa (pp. 197-214). Huelva: Universidad de Huelva.

Massa, E., Pat, M., Keb, A., Canto, V., \& Chan, N. (2011). La definición de amor y dependencia emocional en adolescentes de Mérida, Yucatán. Revista Electrónica de Psicología Iztacala, 14(3), 176-193. 
Méndez, Z., Favila, M., Valencia, A., \& DíazLoving, R. (2012). Adaptación y propiedades psicométricas del cuestionario de dependencia emocional pasiva en una muestra mexicana. Nueva Época, 9(19), 113-128.

Mejía, R. (2010). Una vivencia para prevenir la violencia en el noviazgo: Taller de sensibilización para ser aplicado por facilitadores (Tesina maestría inédita). Universidad Pedagógica Nacional, México.

Montero, M. (2009). Taller: Luchando contra la dependencia emocional en las parejas. Una propuesta (Tesis de licenciatura inédita). Universidad Nacional Autónoma de México, México.

Olvera, A., Arias, J., \& Amador, R. (2012). Tipos de violencia en el noviazgo: Estudiantes universitarias de la UAEM, Zumpango. Revista Electrónica de Psicología Iztacala, 15(1), 150-170.

Organización Mundial de la Salud [OMS]. (2011). Prevención de la violencia sexual y violencia infligida por la pareja contra las mujeres: Qué hacer y cómo obtener evidencias. Washington: Organización Panamericana de la Salud.

OMS. (2012). Centro de prensa. Recuperado de http://www.who.int/mediacentre/factsheets/ fs $239 / \mathrm{es} /$

Oropeza, R. (2011). Dependencia emocional, violencia y satisfacción marital en la relación de pareja (Tesis de licenciatura inédita). Universidad Nacional Autónoma de México, México.
Pérez, R. (2012). Factores psicológicos que desencadenan la violencia hacia la mujer durante el noviazgo (Tesina de licenciatura inédita). Universidad Nacional Autónoma de México, México.

Ramírez-Rivera, C. A., \& Núñez-Luna, D. A. (2010). Violencia en la relación de noviazgo en jóvenes universitarios: Un estudio exploratorio. Enseñanza e Investigación en Psicología, 15(2), 273-283.

Rivera, S., \& Díaz-Loving, R. (2002). La cultura del poder en la pareja. México: UNAM. Rojas-Solís, L. (2013). Violencia en el noviazgo y sociedad mexicana posmoderna: Algunos apuntes sobre la figura del agresor y las agresiones bidireccionales. Uaricha, 10(22), 1-19.

Rubio-Garay, F., López-González, A., Saúl, L., \& Sánchez-Elvira-Paniagua, A. (2012). Direccionalidad y expresión de la violencia en las relaciones de noviazgo de los jóvenes. Acción Psicológica, 9(1), 61-70.

Sarason, I., \& Sarason, B. (2006). Psicopatología. Psicología anormal: El problema de la conducta inadaptada. México: Pearson Educación.

Secretaría de Seguridad Pública [SSP]. (2012). Prevención de la Violencia en el Noviazgo. México: Autor.

Tiscareño, G., \& Velázquez, R. (2011). Dependencia emocional y satisfacción en el noviazgo (Tesis de licenciatura inédita). Universidad Nacional Autónoma de México, México. 